Table 1. Real-Time RT-PCR Analysis of Environmental Samples

\begin{tabular}{|lll}
\hline Location & \multicolumn{1}{c}{ RT-PCR (No. of Samples) } \\
\hline Patient room A & SARS-CoV-2 & Human RPP30 \\
\hline Light switch & Negative $(0 / 1)$ & Negative $(0 / 1)$ \\
\hline Nurse call attached to the bed & Negative $(0 / 1)$ & Positive $(1 / 1)$ \\
\hline Toilet door handle & Negative $(0 / 1)$ & Negative $(0 / 1)$ \\
\hline Bed guard & Negative $(0 / 1)$ & Positive $(1 / 1)$ \\
\hline Anterior room A & & \\
\hline Dust box & Negative $(0 / 1)$ & Positive $(1 / 1)$ \\
\hline Patient room B & & \\
\hline Bed desk & Negative $(0 / 1)$ & Negative $(0 / 1)$ \\
\hline Bed guard & Negative $(0 / 1)$ & Negative $(0 / 1)$ \\
\hline Door handle & Negative $(0 / 1)$ & Positive $(1 / 1)$ \\
\hline Dust box, room side & Negative $(0 / 1)$ & Negative $(0 / 1)$ \\
\hline Dust box, corridor & Negative $(0 / 1)$ & Positive $(1 / 1)$ \\
\hline Control panel on mechanical \\
ventilation & Negative $(0 / 1)$ & Positive $(1 / 1)$ \\
\hline Light switch & & \\
\hline Nurse call & Negative $(0 / 1)$ & Negative $(0 / 1)$ \\
\hline Hand soap dispenser & Negative $(0 / 1)$ & Positive $(1 / 1)$ \\
\hline Anterior room B & Negative $(0 / 1)$ & Negative $(0 / 1)$ \\
\hline Sink, external rim and & & Positive $(1 / 1)$ \\
\hline internal bowl & Negative $(0 / 1)$ & \\
\hline
\end{tabular}

Note. PCR, polymerase chain reaction; RPP30, ribonuclease P 30 subunit; SARS-CoV-2, severe acute respiratory syndrome coronavirus 2 .
Acknowledgments. We thank all of the medical and ancillary hospital staff and the patients for consenting to participate. We thank Suzanne Leech, $\mathrm{PhD}$, from the Edanz Group for editing a draft of this manuscript.

Financial support. This study was supported by a Grant-in-Aid for the Genome Research Project from Yamanashi Prefecture (to M.O. and Y.H.), the Japan Society for the Promotion of Science (JSPS) KAKENHI EarlyCareer Scientists (grant no. JP18K16292 to Y.H.), a Research Grant for Young Scholars (to Y.H.), the YASUDA Medical Foundation (to Y.H.), the Uehara Memorial Foundation (to Y.H.), and Medical Research Grants from the Takeda Science Foundation (to Y.H.).

Conflicts of interest. All authors report no conflicts of interest relevant to this article.

\section{References}

1. Wang D, Hu B, Hu C, et al. Clinical characteristics of 138 hospitalized patients with 2019 novel coronavirus-infected pneumonia in Wuhan, China. JAMA 2020;323:1061-1069.

2. van Doremalen N, Bushmaker T, Morris D, et al. Aerosol and surface stability of SARS-CoV-2 as compared with SARS-CoV-1. N Engl J Med 2020;382: 1564-1567.

3. Hirotsu Y, Mochizuki H, Omata M. Double-quencher probes improved the detection sensitivity of severe acute respiratory syndrome coronavirus 2 (SARS-CoV-2) by one-step RT-PCR. medRxiv 2020. doi: 10.1101/2020.03. 17.20037903.

4. Wang W, Xu Y, Gao R, Lu R, Han K, Wu G, Tan W. Detection of SARS-CoV-2 in different types of clinical specimens. JAMA 2020. doi: 10.1001/jama.2020.3786.

5. To KK, Tsang OT, Chik-Yan Yip C, et al. Consistent detection of 2019 novel coronavirus in saliva. Clin Infect Dis 2020. doi: 10.1093/cid/ ciaal49.

6. Kampf G, Todt D, Pfaender S, Steinmann E. Persistence of coronaviruses on inanimate surfaces and their inactivation with biocidal agents. J Hosp Infect 2020;104:246-251.

7. Ong SWX, Tan YK, Chia PY, et al. Air, Surface environmental, and personal protective equipment contamination by severe acute respiratory syndrome coronavirus 2 (SARS-CoV-2) from a symptomatic patient. JAMA 2020. doi: 10.1001/jama.2020.3227.

Supplementary material. To view supplementary material for this article please visit https://doi.org/10.1017/ice.2020.144

\title{
COVID-19 affects healthy pediatricians more than pediatric patients
}

\section{Nima Rezaei MD, PhD (1)}

Research Center for Immunodeficiencies, Children's Medical Center, Tehran University of Medical Sciences, Tehran, Iran, Department of Immunology, School of Medicine, Tehran University of Medical Sciences, Tehran, Iran and Network of Immunity in Infection, Malignancy and Autoimmunity (NIIMA), Universal Scientific Education and Research Network (USERN), Tehran, Iran

To the Editor-Coronavirus disease 2019 (COVID-19) emerged in China in late December and has spread rapidly throughout the world. The World Health Organization (WHO) considers it a pandemic. In $\sim 4$ months from start of this outbreak, as of April 4, 2020, $>1,000,000$ patients had been affected. ${ }^{1}$

Meanwhile, children appear less likely to be affected than adults; among the affected children, most have mild symptoms and some are even asymptomatic. ${ }^{2-4}$ The Chinese Center for

Author for correspondence: Nima Rezaei, E-mail: rezaei_nima@tums.ac.ir Cite this article: Rezaei N. (2020). COVID-19 affects healthy pediatricians more than pediatric patients. Infection Control \& Hospital Epidemiology, 41: 1106-1107, https:// doi.org/10.1017/ice.2020.139
Disease Control and Prevention has reported that among $\sim 70,000$ cases, $<1 \%$ were aged $<10$ years. ${ }^{5}$

The first official COVID-19 case in Iran was announced on February 19, 2020. On March 16, 2020, 4 weeks later, the daily situation report on COVID-19 showed that $<1 \%$ of $\sim 5,000$ confirmed cases were children aged $<10$ years. ${ }^{6}$ The Children's Medical Center in Iran has 348 beds for pediatric patients, with bed occupancy rate of $94.7 \%$ and an average length of stay of 3.3 days. During the 4 -week period after the first identified case in the country, only 3 positive COVID-19 cases were identified, for an estimated frequency of 1 per 1,000 admitted pediatric patients. Meanwhile, among 60 pediatricians working in this hospital, 5 were positive for COVID-19 by real-time reverse 
transcription polymerase chain reaction (rRT-PCR), for a prevalence of $8.3 \%$, which was much higher than the frequency of COVID-19 in general population ( 2 per 10,000) during this 4 week period.

One hypothesis is that aging is a condition associated with inflammation, whereas children might have an immature anti-inflammatory response. Possibly, therefore, an increased inflammatory reaction is expected in adult subjects compared to children. ${ }^{7}$

Not only the pediatricians but also other healthcare professionals are at a higher risk of infection with COVID-19. ${ }^{8}$ A report from Italy revealed that $\sim 20 \%$ of healthcare professionals had become infected. ${ }^{9}$ Healthcare professionals are at the frontline of fighting COVID-19 in hospitals, where they are in contact with visiting patients and their parents who are potentially carriers of SARS$\mathrm{CoV}-2$, even if they are not infected. Indeed these healthcare professionals are under excessive workload pressure and psychological distress during the pandemic, which can lead to caregiver burnout. ${ }^{10}$ Pediatricians, especially those who have children at home, not only have concerns about passing the infection to their children but also about not caring enough for their children during quarantine period, considering school closures and social distancing policies. ${ }^{8}$ Therefore, healthcare systems should be very careful to address the physical and mental health of healthcare professionals. Easy access to personal protective equipment, especially for those who are visiting patients with COVID-19, and psychological support for those who are losing their patients and colleagues, especially for those who cannot see their family members for long periods, are necessary.

Acknowledgments. This letter is dedicated to honoring the memory of our brave fallen doctors and nurses who fought against COVID-19.
Financial support. No financial support was provided relevant to this article.

Conflicts of interest. All authors report no conflicts of interest relevant to this article.

\section{References}

1. Johns Hopkins Coronavirus Resource Center website. https://coronavirus. jhu.edu/map.html. Accessed April 4, 2020.

2. Dong Y, Mo X, Hu Y, et al. Epidemiology of COVID-19 among children in China. Pediatrics 2020 March 16. doi: 10.1542/peds.2020-0702.

3. Cruz A, Zeichner S. COVID-19 in children: initial characterization of the pediatric disease. Pediatrics 2020 March 16. doi: 10.1542/peds.2020-0834.

4. Lu X, Zhang L, Du H, et al. SARS-CoV-2 infection in children. N Engl J Med 2020 Mar 18. doi: 10.1056/NEJMc2005073.

5. Wu Z, McGoogan JM. Characteristics of and important lessons from the coronavirus disease 2019 (COVID-19) outbreak in China: summary of a report of 72314 cases from the Chinese Center for Disease Control and Prevention. JAMA 2020 Feb 24. doi: 10.1001/jama.2020.2648.

6. COVID-19 daily epidemiology journal. Iranian Ministry of Health and Medical Education website. http://corona.behdasht.gov.ir/files/sitel/files/Factsheet_4. _26.12_-_En.pdf. Published March 16, 2020. Accessed April 15, 2020.

7. Saghazadeh A, Rezaei N. Immune-epidemiological parameters of the novel coronavirus-a perspective. Expert Rev Clin Immunol 2020 Apr 1. doi: 10. 1080/1744666X.2020.1750954.

8. Lo D. COVID-19: protecting healthcare workers. Lancet 2020;395(10228): 922. doi: 10.1016/S0140-6736(20)30644-9.

9. Remuzzi A, Remuzzi G. COVID-19 and Italy: what next? Lancet 2020 Mar 13. doi: 10.1016/S0140-6736(20)30627-9.

10. Moazzami B, Razavi-Khorasani N, Dooghaie Moghadam A, Farokhi E, Rezaei N. COVID-19 and telemedicine: immediate action required for maintaining healthcare providers well-being. J Clin Virol 2020 Apr 4. doi: $10.1016 /$ j.jcv.2020.104345.

\title{
An easy protective measure in ophthalmology against medical supply shortage
}

\author{
Fen Tang ${ }^{1,2}$, Guangyi Huang ${ }^{1}$, Wei Huang ${ }^{1}$ and Fan Xu MD ${ }^{1}$ (1) \\ ${ }^{1}$ Department of Ophthalmology, People's Hospital of Guangxi Zhuang Autonomous Region, Nanning, Guangxi, China and ${ }^{2}$ Department of Ophthalmology, \\ Renmin Hospital of Wuhan University, Wuhan, Hubei, China
}

To the Editor-The rapidly spreading pandemic of coronavirus disease 2019 (COVID-19) poses a huge challenge to global public health. Healthcare systems in many countries are facing severe shortages of medical supplies. ${ }^{1-3}$

Healthcare workers in ophthalmology departments are susceptible to cross infection because routine ophthalmic examinations, such as like slit-lamp microscopy, are usually performed in a setting of close doctor-patient contact. ${ }^{4}$ Three ophthalmologists in Wuhan Central Hospital were infected with SARS-CoV-2 and died from severe COVID-19., At the ophthalmic center of Guangxi

Author for correspondence: Fan Xu, E-mail: oph_fan@163.com

Cite this article: Tang F, et al. (2020). An easy protective measure in ophthalmology against medical supply shortage. Infection Control \& Hospital Epidemiology, 41: 1107-1108, https://doi.org/10.1017/ice.2020.136
Province, we have continued to provide medical services for outpatients who need timely services during the COVID-19 pandemic. However, in response to the lack of personal protective equipment (PPE) (eg, face shields and gowns) during the epidemic peak in China, ${ }^{7}$ we have adopted an easy measure to perform slitlamp examinations. After thorough disinfection, we installed a home-made shield at the slit-lamp to separate patients and ophthalmologists. As shown in Figure 1, the protective shield is placed between the chin rest/headrest and the microscope.

The protective shield could prevent contamination of the slitlamp by respiratory droplets. Although the shortage of medical supplies is currently being alleviated in China, we are encouraging the continuation of this easy protective measure to curb cross transmission in ophthalmology and to protect the ophthalmologists on the frontline of the pandemic. 